\title{
Persistence and integration: Two consequences of a sliding integrator
}

\author{
M. J. PENNER \\ Hunter College of the City University of New York, New York, New York 10021
}

\begin{abstract}
The detection of a silent interval, or gap, placed in the temporal center of a gated noise burst was investigated. The gated noise masker ranged from 2 to $400 \mathrm{msec}$ in duration. For long noises, the duration, $\Delta$, of the just-detectable gap remained fixed at about $2.8 \mathrm{msec}$. Progressively shortening the duration of the noise did not affect $\Delta$ until the duration was approximately $20 \mathrm{msec}$; thereafter, decreasing the noise duration improved detectability of the gap. In a second experiment, continuous noise filled the temporal gap, although the decibel difference between the noise in the gap and the noise surrounding the gap was always at least $5 \mathrm{~dB}$. The level of noise filling the gap did not greatly affect $\Delta$. The third experiment was similar to the first, except that the signal was a click rather than a gap. The results for both gaps and clicks were fitted by a model assuming a sliding integrator.
\end{abstract}

The notion of persistence refers to the observation that the internal representation of a sensory stimulus outlasts its physical presence. Much data supports this view, at least in rough form (e.g., see Sperling, 1960 , for work in vision, and Plomp, 1964, for work in audition). Temporal integration, another wellestablished hypothesis, holds that the statistic governing the detection of a signal in noise results from the summation of sensory stimulation for a period of time. The evidence favoring this view comes from studies showing a reciprocal relation of duration and intensity for threshold detection of brief stimuli. In vision, these results are known as Bloch's law, and a time-intensity trade has also been found in audition (Garner \& Miller, 1947).

The two hypotheses of persistence and integration have often served as a base for models of sensory phenomena. For example, the detectability of a gap in the temporal center of a noise is thought to be limited by the persistence of the first noise (Plomp, 1964), whereas the detectability of a click in the temporal center of a noise is thought to be limited by the duration of an integrator (Penner, Robinson, \& Green, 1972). Thus, integration processes have been used to explain the detection of clicks in noise and the hypothesis of sensory persistence has been used to explain the detection of gaps in noise. In integration models, persistence of information is needed in order for integration to occur. On the other hand, in persistence models, integration is needed to explain the failure of gap detection. It seems reasonable, therefore, to develop a single model utilizing these two concepts to explain both gap and click detection.

This research was supported in part by a grant from the National Institute of Health and in part by a Faculty Research Award $(10772 N)$ from the City University of New York. I would like to thank H. Duifhuis and B. Leshowitz for their helpful comments on a previous version of the manuscript.
To this end, three experiments were undertaken. In the first experiment, the detectability of a gap temporally placed in the center of a noise masker is tracked as a function of the duration of the noise masker. In the second experiment, various levels of noise filled the gap. For each noise level, the duration of the gap was varied to find the threshold. The third experiment was similar to our first experiment, except that clicks rather than gaps were used. The results of these three experiments provide data which can be used to formulate a single mechanism whose properties result in both persistence and integration.

\section{METHODS}

\footnotetext{
Apparatus

Gaps. The durations of the noises and the gap between them were controlled by a Tempo computer. The noise was generated by a random noise generator (General Radio Model 1382), and was led to an electronic switch (Grason-Stadler Model 1287) and then filtered by two cascaded Allisons (Model 2A, bandwidth 0 to $10 \mathrm{KH}$ ) , and finally served as the binaural input to two TDH-49 headphones. In the tirst experiment, the noise spectrum level was either 28 or $48 \mathrm{~dB} \mathrm{SPL}$ and the combined duration of the two noises bounding the gap ranged from 2 to $400 \mathrm{msec}$. For the second experiment, the gated noise was either 6 or $60 \mathrm{msec}$. In this experiment, continuous uncorrelated noise was simply added to the input to the headphones. The decibel difference between the continuous and gated noise, the interburst level, ranged from $-\infty$ (silence) to $-5 \mathrm{~dB}$, but the noise spectrum level of the gated noise was adjusted to remain at $28 \mathrm{~dB}$ SPL.

Clicks. The signal, a $100-\mu$ sec click, was generated by a pulse generator (General Radio, 1217-B). Its spectrum was essentially flat up to $5.000 \mathrm{~Hz}$. The click was measured by repeating it periodically and using a wave analyzer (General Radio, Model 1900A) to measure the voltage of the click train in specified frequency regions (see Penner et al., 1972). The click was added to the noise, and the complex was passed through a pair of cascaded filters (Allison Model 2A. Universal Model 1952: bandpass set for $100-5,000 \mathrm{~Hz}$ ). Stimuli were presented binaurally over TDH-49 headphones. The experiment was controlled by a Tempo computer. The noise was generated by a General Radio (1382) random noise generator and gated on by an electronic switch (Grason Stadler Model 1287).
} 


\section{Procedure}

In the first experiment. the duration of the just detectable gap. $\Delta$. was measured as a function of the duration of the surrounding gated noise. The combined duration of the noise bounding the gap was examined for nine durations, ranging from 2 to $400 \mathrm{msec}$. For one of the two subjects. L.P.. data were obtained for only one noise level. $\mathrm{N}_{\mathrm{O}}=28 \mathrm{~dB}$ SPL. For the second subject. M.J.P., data were obtained for two noise levels. $\mathrm{N}_{\mathrm{o}}=28$ and $\mathrm{N}_{\mathrm{o}}=48 \mathrm{~dB}$ SPL. In the second experiment. $\Delta$ was measured as a function of the level of the continuous noise filling the gap. A total of seven levels was used, the interburst level ranging from $\infty$ to $-5 \mathrm{~dB}$. The gated noise was fixed at either 6 or $60 \mathrm{msec}$. In the third experiment, the threshold of the click. $E_{C}$. in noise was measured as a function of the surrounding noise duration. which ranged from 0.14 to $600 \mathrm{msec}$. In the three experiments. the signal thresholds were determined in the following ways.

Experiment I. Binaural thresholds were determined using a two-interval temporal forced-choice procedure in which the interval containing the gap was arbitrarily designated as correct. During a dailv session. all noise durations were presented in haphazard order. Two psychophysical techniques were employed. In the first experiment. a standard six-step up-down procedure was used to measure the detectability of the gap. That is. the interval between the noises was initially fixed at $T$ msec and decreased in decrements of $\Delta T$ until a gap of $T-5 \Delta T$ was obtained. whereupon in the next six trials the gap systematically increased to $T$, and so forth. Using this procedure, two psychometric functions were determined. Each function consisted of 600 total observations with 100 observations for each value of $\Delta \mathrm{T}$.

Experiments II and III. In the second and third experiments, a tracking procedure was used to determine threshold. Threshold is defined as the value of $\Delta T$ (or $E_{c}$. click energy) necessary for $71 \%$ correct discrimination. The procedure is as follow's: given two successive correct responses. $\Delta T$ (or $E_{c}$ ) was decreased; given one incorrect response. $\Delta T$ (or $E_{c}$ ) was increased: otherwise, $\Delta T$ (or $E_{c}$ ) was not changed. The first 30 trials of each block of 130 trials were ignored: only the last 100 trials were averaged to provide a measure of the threshold. Four hundred observations determined each point (see Levitt, 1970. for procedural details).

The author served as a subject in all experiments. There were two additional subjects. one serving in the gap- and one in the click-detection experiments.

\section{RESULTS}

\section{Experiment I}

Figure 1 shows the effect of the gated noise duration on the detection of a gap for two noise levels. The ordinate is the gap duration, $\Delta$, necessary for $75 \%$ correct discrimination. The abscissa is the combined duration of the two noise bursts bounding the gap. The data in Figure 1 can be roughly described with two intersecting straight lines. One line has a 0 -msec slope and fits the data for noise durations exceeding $20 \mathrm{msec}$. The other line has a slope of $2 \mathrm{msec}$ [i.e.. $\Delta=2.0 \mathrm{msec} \times \log$ (noise duration) + constant] and is obtained for noises less than $20 \mathrm{msec}$. The line with 0 -msec slope indicates that a change in noise masker duration does not affect detection. The line with $2-\mathrm{msec}$ slope indicates that decreasing the noise masker duration improves detection.

There are two points of note from these results. First, in a similar paradigm, with long-duration gated noises. Plomp (1964) finds that the detectable gap ranges from 2.3 to $3.5 \mathrm{msec}$. Our results replicate his. Furthermore, in both Plomp's study and ours, as $\mathrm{N}_{\mathrm{O}}$ increased. $\Delta$ also increased. Second, it might be argued that decreasing the noise duration improves detection because. for brief durations, the subjects based their discrimination on the total duration and selected the interval which appeared longest. However. the detectable gaps are considerably briefer than would have been the case had discriminations been based on total duration (Abel, 1972). Finally. even though both subjects reported picking the louder
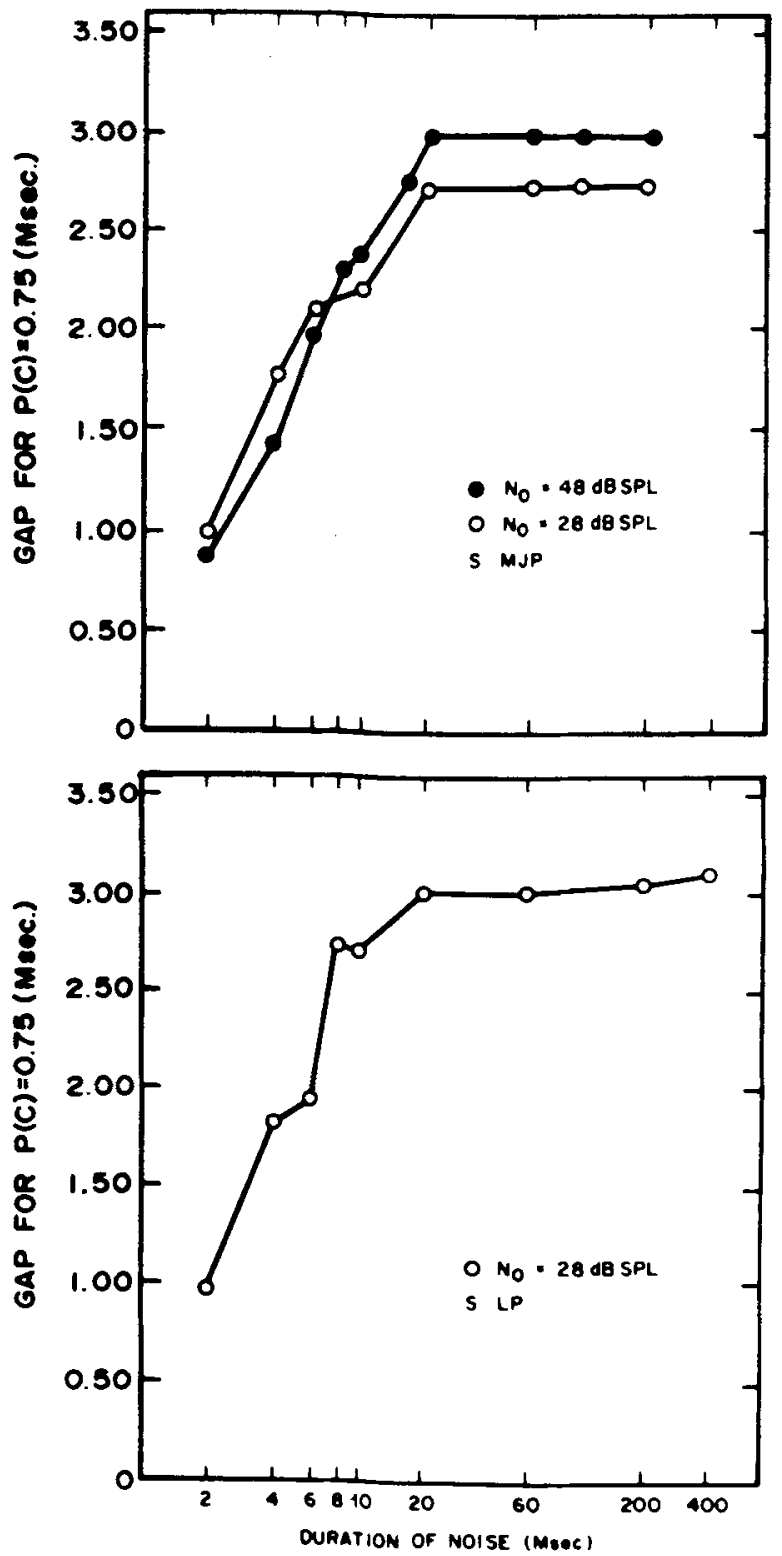

Figure 1. The effect of masker duration on the gap threshold. On the ordinate is the gap duration required for $75 \%$ detection. On the abscissa is the duration of the noise masker. The noise spectrum level is either $48 \mathrm{~dB}$ SPL (the Xs) or $28 \mathrm{~dB}$ SPL (circles). The upper and lower panels represent the two subjects' data. 

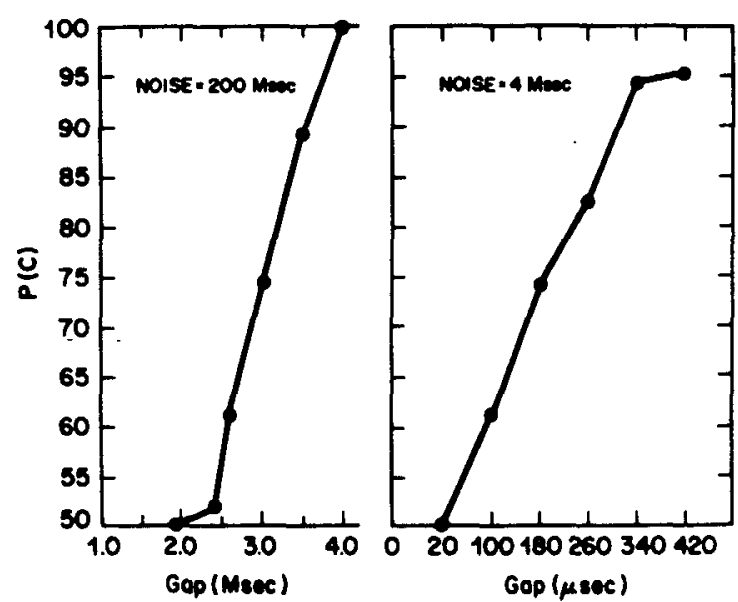

Figure 2. Psychometric function for the gap in the temporal center of the noise. On the left is the psychometric function for a 200-msec noise masker. On the right is the psychometric function for a 4-msec noise masker. Functions are for M.J.P. only.
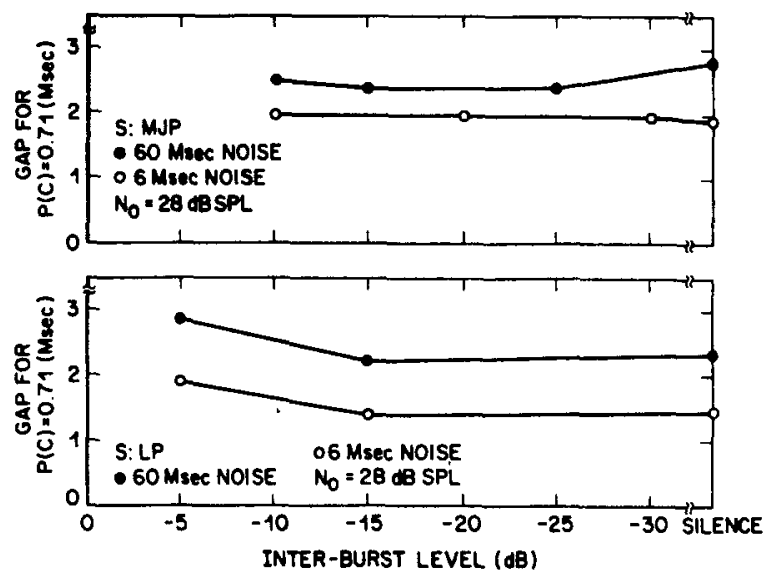

Figure 3. The effect of interburst level on the gap. On the ordinate is the gap duration required for $71 \%$ correct detection. On the abscissa is the interburst level, the decibel difference between the gated and continuous noise. The dots represent a 60 -msec noise masker, and the circles represent a 6-msec noise masker. The upper and lower panels represent the two subjects.

stimuli at the brief duration, it must be noted that the two comparison stimuli were of equal total energy, so that any difference in loudness must be attributed to the presence of the gap.

Figure 2 shows the psychometric functions associated with the $4-\left(N_{O}=28 \mathrm{~dB} \mathrm{SPL}\right)$ and $200-\left(\mathrm{N}_{\mathrm{O}}\right.$ $=48 \mathrm{~dB}$ SPL) msec noise maskers for the author, M.J.P. There are 200 observations per point.

\section{Experiment II}

Figure 3 shows the effect of the interburst level on the detectability of the gap for two noise durations, 60 and $6 \mathrm{msec}$. The interburst level (Pollack, 1954) is defined as the $\mathrm{dB}$ difference between the noise filling the gap and the noise surrounding it. Note that the interburst level differs only by a constant from the level of noise in the gap. The ordinate in Figure 3 is the gap duration necessary for $70.7 \%$ (rounded to 0.71 in the figure) discrimination. The abscissa is the interburst level.

The data in Figure 3 are well fitted by a linear function with a slope of about zero $(\mathrm{msec} / \mathrm{dB}$ ), indicating that, for a wide range of interburst levels, detectability is independent of the interburst level. Of course, the threshold must rise as the interburst level approaches zero since, at that point, there would be no gap. The available equipment did not allow us to examine interburst levels between -5 and $0 \mathrm{~dB}$. The interesting point to note, however, is that detectability did not change, even with the interburst levels investigated. This result is supported by Pollack's (1954) study of masking by a periodically interrupted noise. Pollack finds that the threshold of a tone is unaffected by the interburst level (as long as the noise in the gap is less than or equal to the surrounding noise, and as long as the gap is 3 msec or less).

\section{Experiment III}

Figure 4 shows the effect of noise duration on the detection of a click in the temporal center of the noise. The ordinate is the ratio of the click energy to the noise energy in a one-cycle band necessary for $70.7 \%$ correct detection. The abscissa is the duration of the noise surrounding the gap. These data are in good agreement with the previous results (Penner \& Cudahy, 1973; Penner et al., 1972). Both sets of previous results used an up-down procedure, whereas the present task was an adaptive procedure so that a comparison with our gap data could be made easily. The line through the solid circles represents a theoretical fit to the data which will be discussed in more detail in a later section.

Note that Experiment III is not directly comparable

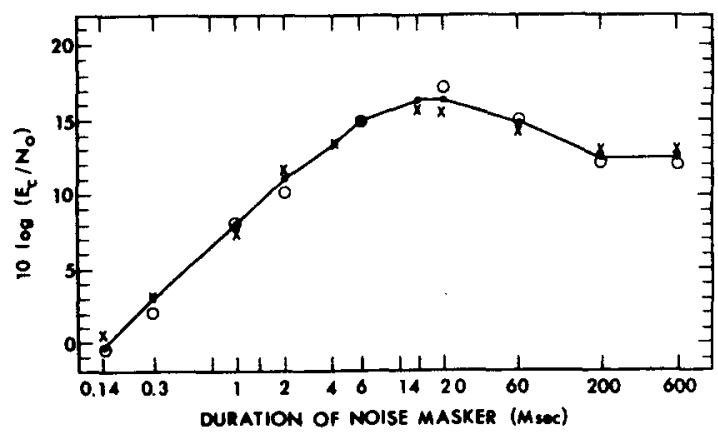

Figure 4. Effect of masker duration on the click threshold. The passband of the signal and masker is 100 to $5,000 \mathrm{~Hz}$. The noise spectrum level, $\mathbf{N}_{0}$, is $36 \mathrm{~dB}$ SPL. The abscissa is the noise duration, the ordinate is the ratio of the click energy to the noise energy in a one-cycle band. The $X_{s}$ represent the data of M.J.P. (500 observations per point), and the empty circles represent the data of R.S. ( 300 observations per point). The filled circles are the theoretical predictions of the model. 
to Experiment I. In Experiment I the gap duration is varied in order to reach threshold, whereas in Experiment III the click energy (for a constant duration) is varied in order to reach threshold. In Experiment II. both the gap duration and the energy in the gap are varied, and theoretically, then, Experiment II provided a bridge between the two procedures. but the equipment limitations precluding interburst levels below $5 \mathrm{~dB}$ did not allow extrapolations to be made directly. Nevertheless, all these points being admitted, there is still every reason to believe that a single model could be found to deal with the data from these three experiments.

\section{DISCUSSION}

Over the years. the energy detection model (Green d Swets. 1966) has received a good deal of support from masking experiments employing brief signals and wide-band maskers. Basically, it predicts that for brief signals (certainly for the brief click and gap durations in our study) detectability is determined by the ratio of the signal energy to the noise power density. Applying this notion to the third experiment. He observe that the signal-to-noise ratio necessary to detect a click in the center of a 400-msec noise masker is about $12 \mathrm{~dB}$ (for calculational details, see Penner et al.. 1972). By calculating the energy that would have been present in the gap. had the noise remained on. a similar signal-to-noise ratio may be calculated for the gap signal in Experiment I. The signal-to-noise ratio necessary to detect a gap in the center of a $400-\mathrm{msec}$ noise masker is $18 \mathrm{~dB}$. Calculations for other noise durations indicate that, in general. a smaller signal-to-noise ratio results in detection of clicks than of gaps.

One reason for this discrepancy may be that energy detection models do not incorporate any notion of persistence. To see that persistence might be a key factor. note that the signal-to-noise ratio for gaps is calculated from the energy missing in the physical gap. Although the stimulus may not be physically present during the gap, the auditory stimulus's response may continue relatively unabated, thereby making the internal signal-to-noise ratio substantially smaller than the physical ratio. This argument can be applied to the click as well, but an increase in the persistence of a click should not cause detectability to decrease.

To sum up, then. an appropriate formulation of the energy detection model holds that the physical signal-to-noise ratio determines detection. However, our argument holds that at short signal durations, the internal and physical signal-to-noise ratios do not match. Therefore, it is only for long signal durations that we can expect the energy detection model to apply to both increments and decrements. By long, we mean a signal whose physical duration is long relative to its internal persistence. The main point. for the present argument. is that classical energy detection models cannot account for the detection of both gaps and clicks: a new direction is necessary.

\section{The Running Average Model: A Sliding Integrator}

One approach to modeling the data in this study is provided by the running-average model of Munson (1947) and Zuisiocki (1969). The central idea in this model is that detection of a signal is based on the output of a short-term averaging mechanism whose properties are essential in specifying the subject's internal representation of a stimulus. Let $w(t)$ be the averaging device, $x(t)$ the input wave form to the averager. and $y(t)$ the quantity upon which the subject bases his response. The averaging assumption relates these quantities as follow's:

$$
y(t)=\int_{0}^{\infty} w(\tau) x(t-\tau) d \tau
$$

The duration of $w(t)$ represen ${ }^{+c}$ "integration time." and the time course of the decay of $y(t)$ represents persistence. In order to apply this sliding integrator. $w(t)$. to an experimental setting, it is necessary to specify both $y(t)$. the subject's internal representation of the stimulus. and a decision rule which governs the subject's responses. $y(t)$ is determined solely by the physical input, $x(t)$, and the weighting function, $w(t)$, whose form must be assumed.

The shape of $w(t)$ is crucial in that it determines $y(t)$ and the way in which the model encompasses persistence and integration. If $w(t)$ is chosen so that v(l) gradually decays to zero, then stimuli will persist after their physical termination. Further, the duration of $w(t)$ determines integration time. In the following sections, these notions are made mathematically explicit by applying them to the data for gap and click detection experiments,

\section{Gap Detection}

Consider first the decision process: the property of $y(t)$ that is likely to be utilized in detecting a gap in Experiment I. Figure 5 shows $y(t)$ for two noise durations. $w(t)$ is assumed to be exponential (Duithuis. 1973).

$$
w(t)=e^{-t / \xi} \quad t \geqslant 0
$$

and $\xi$ is the time constant of the integrator. In the upper panel, the gated noise duration is much longer than the time constant of the integrator. In the lower panel. the duration of each burst is much briefer than the time constant of the integrator. An obvious difference is immediately apparent. For long noise durations, $y(t)$ reaches a steady state value in the interval containing the gap. This suggests that the subject may detect the gap by noting a decrease in $y(t)$ within a single interval. For brief noise durations, on the other hand. $y(t)$ does not reach a steady value in either interval. As is diagrammed. $y(t)$ actually rises to a larger value in the interval without the gap. This 
suggests that the subject may detect the gap by comparing the values of $y(t)$ in the two intervals.

One decision rule that bases detection on comparisons of $\mathrm{y}(\mathrm{t})$ between the two intervals or within a single interval is a ratio rule basing the detection decision on the ratio of the maximum value of $y(t)$ to the minimum value of $y(t)$ [given that $y(t)$ has increased from zero input]. As seen in Figure 5, the local minimum always occurs at time $T_{2}$ and the maximum always occurs at $T_{3}-\Delta$. For long noises, the maximum value of $y(t)$ occurs at time $T_{3}-\Delta$ and is the same as the value of $y(t)$ at numerous other times. Thus, even in a 2 IFC procedure, the decision rule does not necessitate a comparison of the values of $y(t)$ between the two intervals of the forced choice procedure. For brief noises, the maximum value of $y(t)$ occurs only at time $T_{3}-\Delta$. Thus, for brief noises, the decision rule necessitates a comparison of the values of $y(t)$ between the two intervals. For these brief noises, at time $T_{3}-\Delta, y(t)$ is larger for the interval without the gap. Using the ratio of the $y(t) s$ as the detection statistic, we now proceed with formalizing the model.

For the interval with the gap, the function $x(t)$ may be described as follows (see Figure 5):

$$
\begin{array}{ll}
x(t)=A & 0 \leqslant t \leqslant T_{1} \\
x(t)=0 & T_{1}<t<T_{2} \\
x(t)=A & T_{2} \leqslant t \leqslant T_{3},
\end{array}
$$

and for the interval without the gap,

$$
x(t)=A \quad 0 \leqslant t \leqslant T_{3}-\Delta,
$$

where $T_{2}-T_{1}=\Delta$.

The quantity upon which the subject bases his decisions is assumed to be the ratio of the maximum value of $y(t), y_{\max }$, to the minimum value of $y(t)$, $y_{\min }$. For the interval without the gap, $y_{\max }$ is the value of $y(t)$ at time $T_{3}-\Delta$. For the interval containing the gap, $y_{\min }$ occurs at time $T_{2}$ so that

$$
\frac{y_{\max }}{y_{\min }}=\frac{A\left[1-e^{-\left(T_{3}-\Delta\right) / \xi}\right]}{A\left[1-e^{-\left(T_{1} / \xi\right)}\right]\left[e^{-\Delta / \xi}\right]} .
$$

Assume that a similar ratio is constructed by the subject in which $y_{\max }$ is taken from the interval with the gap and $y_{\min }$ is taken from the interval without the gap. The subject will attempt to choose the larger of these two ratios. However, we assume internal noise in this comparison process so that errors are made more often when the ratios become more nearly equal. Finally, then, there is a particular value of the ratio in Equation 7 which will correspond to a $75 \%$ correct choice during this comparison process (i.e., to threshold).

Equation 7 can be easily understood. It represents
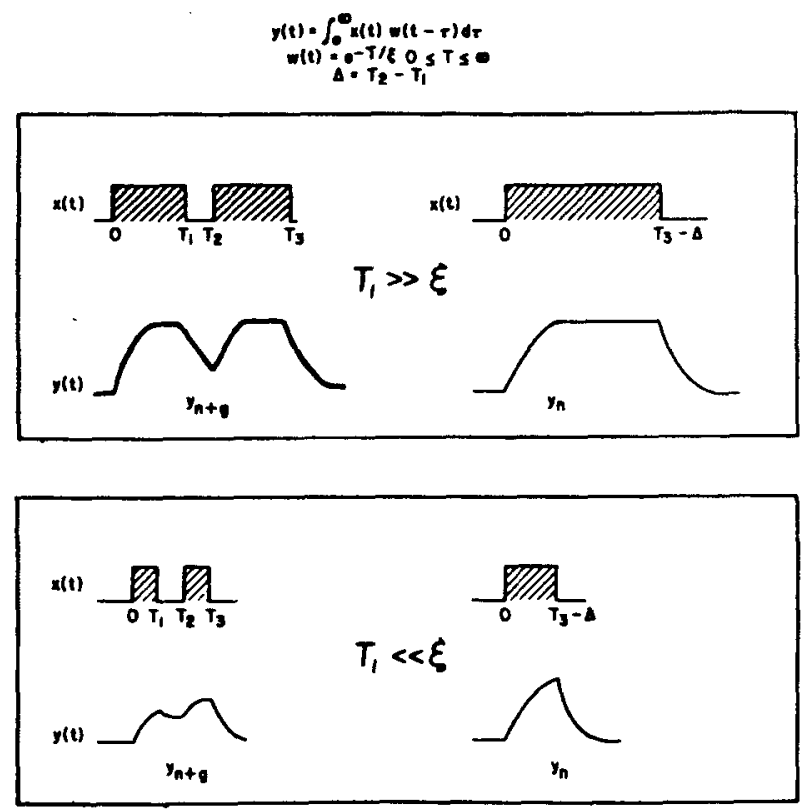

Figure 5. Schematic representation of the basic idea. The noise bursts are depicted as $x(t)$, and the output of the integrator is $y(t)$. The integrator, $w(t)$, is assumed to be exponential. Appropriate sketches of $y(t)$ for very long and very brief noise bursts are drawn.

two processes. The denominator represents persistence, the decay of sensation; it is the residual value of $y(t)$ resulting from the first noise after a decay lasting $\Delta$ msec. The numerator represents integration, the rise of sensation; it is the maximum level of $y(t)$ resulting from continuous stimulation with the two noise bursts. Since the decay process proceeds for long or short noises, it is the rise represented by the numerator that is principally responsible for the improvement in the detectability of the gap as the noise duration decreases. This is easily seen by noting that, for long noises, the maximum level of $y(t)$ is larger at the end of the second noise than at the end of the first noise. It is this circumstance that leads to the increased detectability of the gap as the noise duration decreases.

Let us now use Equation 7 to fit the data in Figure 1. The values of $y_{\max } / y_{\min }$ and $\xi$ which minimize the sum of squared deviations of the prediction from the data in Figure 1 are: For M.J.P., $\xi=2.25 \mathrm{msec}$ and $\mathrm{y}_{\max } / \mathrm{y}_{\min }=3.7$ for $\mathrm{N}_{\mathrm{o}}=48 \mathrm{~dB}$ SPL; $\xi=3.04 \mathrm{msec}$ and $\mathrm{y}_{\max } / \mathrm{y}_{\min }=2.47$ for $\mathrm{N}_{\mathrm{O}}$ $=28 \mathrm{~dB}$ SPL; and for L.P., $\xi=3.5$ and $\mathrm{y}_{\max } / \mathrm{y}_{\min }$ $=2.40$ for $N_{0}=28 \mathrm{~dB}$ SPL. The values of $\xi$ are in near agreement with Diufhius' (1973) value of 2 msec. The value of the ratio $y_{\max } / y_{\min }$ appears to lie between 2 and 4 , as does the value of the corresponding parameter in Zwislocki's (1969) fit to his data data on the temporal summation of loudness. In both models, the ratio reflects the subject's sensitivity.

Finally, consider the effect of adding noise to the gap. If $y_{\max } / y_{\min }=2$, then a $3-\mathrm{dB}$ decrement is 
detectable. If the first noise burst has a spectrum level of $N_{0}$, the gap threshold is unaffected until the intermediate level is $-3 \mathrm{~dB}$. This conforms to our finding that essentially no threshold shift occurred for interburst levels exceeding $-5 \mathrm{~dB}$.

\section{Click Detection}

The sliding integrator can also be used to account for the detection of clicks in the temporal center of noise bursts (lasting $\mathrm{T}_{3}$ msec). We adopt the same decision rule as was utilized for detection of gaps. The response to the masker alone, $y_{m}(t)$, computed from Equation 3 using an exponential integrator, is a maximum at $T_{3}$ and is

$$
y_{m}(t)=A\left(1-e^{-T_{3} / \xi}\right) .
$$

The response to both masker and click is a maximum at shortly after time $T_{3} / 2$ and is

$$
y_{c}(t)=A\left(1-e^{-T_{3} / 2 \xi}\right)+B,
$$

where $\mathrm{A}$ and $\mathrm{B}$ are proportional to the noise and click amplitudes. If the ratio, $y_{m}(t) / y_{c}(t)=K$, determines detection and if $B / A$ is proportional to $E_{c} / N_{0}$, then

$$
\begin{aligned}
10 \log _{10}\left(\frac{E_{c}}{N_{o}}\right)= & 10 \log _{10}\left(\frac{B}{A}\right) \\
= & 10 \log _{10}-\left\{\left[K\left(1+e^{-T_{3} / 2 \xi}\right)-1\right]\right. \\
& \left.\cdot\left[1-e^{-T_{3} / 2 \xi}\right]\right\}+c
\end{aligned}
$$

where $c$ is a scaling constant, $E_{c}$ the click energy, and $\mathrm{N}_{\mathrm{o}}$ the noise power per cycle per second. For small $\mathrm{T}_{3} / \xi$,

$$
\begin{aligned}
& 10 \log _{10}\left(\frac{E_{c}}{N_{o}}\right) \\
& =10 \log _{10}\left[(2 K-1) \frac{T}{2 \tau}\right]+c \quad T_{3} \ll \xi .
\end{aligned}
$$

For large $T_{3} / \xi$,

$$
10 \log _{10}\left(\frac{E_{c}}{N_{o}}\right)=10 \log _{10}(K-1)+c . T_{3}>\xi .
$$

In order to use the model to predict the form of the function relating $10 \log _{10}\left(E_{c} / N_{o}\right)$ to $T_{3}$, two parameters are needed: $\xi$ and $K$. The remaining parameter, c, merely raises or lowers the entire function. The average values of $\xi$ and $K$ that best fit the click data in Figure 4 are $14 \mathrm{msec}$ and 1.12, respectively, with c equal to $10 \mathrm{~dB}$. This theoretical fit to the data from the click detection study is plotted in Figure 4 . The empty circles and the $x$ are the data for two subjects, and the filled circles and the line through them represent the theoretical fit. The difference in the click threshold for the long $(200 \mathrm{msec})$ and intermediate $(20 \mathrm{msec})$ noise durations has been called overshoot. Note that the $41 / 2-d B$ overshoot seen in Figure 4 is predicted by the model. In general, the size of the overshoot is determined by the value of $K$.

It should be noted that this model predicts that the threshold of a click will depend on its temporal position within the noise. The data show no such dependence (Osman \& Raab, 1963; Penner \& Cudahy, 1973). Some process not taken into account in our simple version of the model may be needed to deal with this case. For example, the criterion, K, may depend on the tomporal position of the click in the noise.

\section{General Discussion}

The parameters estimated from the fits of the model to the data differ for clicks and gaps. The question is why. The discrepancy is not due to the shallowness of the parameter space; an attempt to fit both sets of data with the same parameter values failed badly. A possible source for the discrepancy is suggested by noting that all the above remarks apply only to the decision rule we adopted. A wide variety of other decision rules are possible. It may be that one of these decision rules will result in identical parameter estimates for the click and gap studies. Because this would be a most important result, we are currently exploring alternative rules as well as the effect of representing the response to a noise burst as a stochastic process.

\section{SUMMARY AND CONCLUSION}

The detectability of a gap has traditionally been thought to be limited by the persistence of the first noise (Plomp, 1964). The detectability of a click has been thought to be limited by the duration of an integrator (Penner et al., 1972). In this paper, we have presented a model incorporating a sliding integrator. The sliding integrator is a single mechanism uniting the concepts of persistence and integration. The model has been quantified and successfully fitted to many aspects of the click and gap data. The difference in the parameter estimates for the two types of stimuli may imply that the ear's time constant depends on the stimulus's intensity or that the decision rule picked was not appropriate. Future work will explore other decision rules and alternative models. 


\section{REFERENCES}

ABEL, S. Discrimination of temporal gaps. Joumal of the Acoustical Society of America, 1972, 52, 519-524.

Duifuuts. H. Consequences of peripheral frequency analysis for nonsimultaneous masking. Joumal of the Acoustical Society of America, 1973. 54, 1471-1488.

Garner, W. R.. \& Miller, G. A. The masked threshold of pure tones as a function of duration. Joumal of Experimental Psychology, 1947, 37, 294-303.

Green, D. M., \& Swets, J. A. Signal detection theory and psychophysics. New York: Wiley, 1966.

LEVITT, H. Transformed up-down methods in psychoacoustics. Journal of the Acoustical Society of A merica, 1970, 49, 467-477.

Munson, W. A. The growth of auditory sensation. Journal of the Acoustical Society of A merica, 1947, 19. 584-591.

Osman, E., \& RaAB, D. H. Temporal masking of clicks by noise. Joumal of the Acoustical Society of America, 1963, 46, $965-968$.
Penner, M. J., \& Cudahy, E. The critical masking interval: A temporal analog of the critical band. Journal of the Acoustical Society of America, 1973, 54, 1530-1535.

Penner, M. J., Robinson, C. E., \& Green, D. M. The critical masking interval. Journal of the Acoustical Society of America, 1972, 52, 1661-1669.

PLomp. R. Rate of decay of auditory sensation. Journal of the Acoustical Society of America, 1964, 36, 277-282.

PollaCK. I. Masking by periodically interrupted noise. Journal of the Acoustical Society of America, 1954, 27, 353-355.

SPERLING, $G$. The information available in brief visual presentations. Psychological Monographs, 1960, 7411, Whole No. 498).

Zwislocki, J. J. Tempotal summation of loudness: An analysis. Journal of the Acoustical Society of America, 1969, 46, 431-441.

(Received for publication January 1975; revision accepted A pril 28, 1975.) 